\title{
ANTIOXIDANT AND ANTIMICROBIAL PROPERTIES OF STEVIA LEAVES EXTRACTS AND SILVER NANOPARTICLES COLLOIDS
}

\author{
Iryna Laguta $^{\mathrm{a}^{*}}$, Teteiana Fesenko ${ }^{\mathrm{a}}$, Oksana Stavinskaya ${ }^{\mathrm{a}}$, Oksana Dzjuba ${ }^{\mathrm{b}}$, Lesya Shpak $^{\mathrm{b}}$ \\ ${ }^{a}$ Chuiko Institute of Surface Chemistry of National Academy of Sciences of Ukraine, \\ 17, General Naumov Street, Kiev-03164, Ukraine \\ ${ }^{b}$ M.M. Gryshko National Botanic Garden of National Academy of Sciences of Ukraine, \\ 1, Timiryazevska Street, Kiev-01014, Ukraine \\ *e-mail:icvmtt34@gmail.com
}

\begin{abstract}
Three extracts of Stevia rebaudiana (Bertoni) were prepared using various types of raw materials: leaves of plants grown ex situ, leaves of plants grown in vitro, callus culture formed on damaged leaves. Composition of the extracts, their activity in the synthesis of silver nanoparticles colloids, as well as antioxidant and antimicrobial properties of the extracts and the colloids were investigated.
\end{abstract}

Keywords: Stevia leaves extract, silver nanoparticles colloid, antioxidant activity, antimicrobial properties.

Received: October 2016/ Revised final: November 2016/ Accepted: November 2016

\section{Introduction}

Recently detailed investigation of plants phytoconstituents and extracts has attracted a great attention. A variety of plants was considered as a source of biologically active substances with various valuable properties [1-3]. For example, Acacia arabica was used to extract tannins for the production of natural dyes for textile industry. Atropa belladonna, Nicotiana tabaccum, Cinchona tree were found to contain pharmacologically active substances such as atropine, nicotine, quinine. Madagascar periwinkle was used as a source of anticancer alkaloids. Essential oils extracted from the fruits, flowers, barks, stem, leaves, roots and other parts of various plants are widely used in cosmetic industry and aromatherapy.

For medicine, biology and food industry, bioactive compounds endowed with redox properties, as well as antimicrobial activity, are of especial interest. Plants may be a source of effective antioxidants of natural origin, such as: flavonoids, phenolic acids, phenolic diterpenes, and tannins. All these compounds may be used in pharmacology and cosmetology to produce the preparations with antioxidant, anti-inflammatory, immunomodulation action [1]. Many of plants may also contain such phytoconstituents as terpenoids, alkaloids, coumarins, fatty acids with clear antimicrobial activity [4]. According to the published data, they may be used, in particular, as antimicrobial drugs, antimicrobial agents in food packaging and preservatives in the cosmetic industry [2, 4-7]. An additional way of using the plant extracts with redox properties for medical or biological applications is represented by "green" synthesis of silver nanoparticles (AgNPs), which are known to be very effective antimicrobial agent. Green synthesis of metal nanoparticles (NPs) where plant constituents are employed is also of interest for various fields of science and technologies due to wide application of the NPs in electronics, material science, physics and catalysis [8]. Use of plant extracts for NPs production appears to provide the synthesis with beneficial requirements: mild temperature and $\mathrm{pH}$, non-toxic and cheap reducing/stabilizing agents.

In spite of wide diversity of utilization and application, the extracts of plants grown in the traditional way may also posses certain drawbacks. The formation of metabolites in the plants grown ex situ may be affected by unfavourable ambient conditions; this, in turn, may lead to violation of the synthesis of valuable metabolites and accumulation of toxic substances in the plants. Cultivation of the plant tissues under aseptic conditions is an alternative method for production of pure bioactive compounds. Pureness of bioactive compounds and independence of the metabolites yields on climate conditions and negative ambient effects are the advantages of this method.

The present communication reports on study of the extracts of Stevia rebaudiana (Bertoni) obtained from the leaves of the plants grown ex situ and in vitro as well as from the callus tissue culture formed on damaged leaves. Stevia rebaudiana (Bertoni) is a herbaceous perennial shrub. The Stevia leaves contain a complex mixture of sweet diterpene glycosides [9] as well as such bioactive substances as: flavonoids, phenolic acids, fatty acids, proteins, and vitamins [10]. The availability of compounds with redox properties makes Stevia plants an interesting raw material for preparation of biologically active extracts and for green synthesis of NPs colloids. To the best of our knowledge, comprehensive investigation of these plant extracts from the different types of raw materials as well as the use of such extracts for the green synthesis of metal NPs has not been reported. Our work was also aimed at study of the activity of the Stevia rebaudiana (Bertoni) extracts in synthesis of silver nanoparticles and characterization of the antioxidant/ antimicrobial properties of the extracts and the synthesized colloids.

\section{Experimental}

Stevia plants were grown in M.M. Gryshko National Botanic Garden of National Academy of Sciences of Ukraine. Three types of raw material were used to prepare the Stevia extracts. Extract 1 and Extract 2 were produced 
using leaves of the plants grown ex situ and in vitro, respectively. Extract 3 was prepared from callus. To grow plants in vitro, sterilized seeds were placed in glass flasks containing Murashige and Skoog basal medium and exposed to artificial light for 16 hours per day. To obtain callus, transverse incisions were made on fresh leaves, without reaching the edge of the leaf. Then these leaves were placed on a modified Murashige and Skoog basal medium additionally containing $40 \mathrm{mg} / \mathrm{L}$ of iron(III) chloride and up to $0.2 \mathrm{mg} / \mathrm{L}$ of vitamins B1, B6 and thidiazuron.

The biologically active substances were extracted from the leaves and callus with a $70 \%$ ethanol aqueous solution according to a procedure described in the literature [11]. To $1 \mathrm{~g}$ of finely chopped leaves or callus, $100 \mathrm{~mL}$ of $70 \%$ ethanol were added, then the mixtures were placed into steam bath for 30 minutes. After cooling, the extract was adjusted to the initial volume and filtered.

The total concentration of phenolic compounds in the extracts was evaluated by using the Folin-Ciocalteau method. For measuring the total phenol index [12], $11.5 \mathrm{~mL}$ of distilled water, $5 \mathrm{~mL}$ of $20 \%$ sodium carbonate solution, $1.25 \mathrm{~mL}$ of Folin-Ciocalteau reagent (Merck) and $6.25 \mathrm{~mL}$ of water were consecutively added to $1 \mathrm{~mL}$ of extract. The solution was stirred for $30 \mathrm{~min}$, then the absorbance at $750 \mathrm{~nm}$ was measured, and the total phenol index was calculated in accordance with the described protocol [12]. Using the data on total phenol index for the well known antioxidant, ascorbic acid (for $0.5 \mathrm{mM}$ solution of ascorbic acid the total phenol index was equal to [13]), the equivalent concentration of ascorbic acid in the extracts was calculated.

For determining the total content of flavonoids, we used the method based on the ability of the compounds to form a coloured complex with aluminium chloride. To $1 \mathrm{~mL}$ of extract, prepared as above-described and placed in a volumetric flask, $5 \mathrm{~mL}$ of $2 \%$ solution of $\mathrm{AlCl}_{3}$ (Sigma-Aldrich) in $95 \%$ ethanol was added, and then $95 \%$ ethanol was added to reach the volume of $25 \mathrm{~mL}$. The mixture was stirred for $30 \mathrm{~min}$ and the optical density of the solution at $410 \mathrm{~nm}$ was measured. To prepare a blank solution, $0.1 \mathrm{~mL}$ of concentrated acetic acid was added to $1 \mathrm{~mL}$ of extract, followed by the dilution of the mixture by $95 \%$ ethanol to the total volume of $25 \mathrm{~mL}$. For obtaining the reference samples, rutin (Sigma-Aldrich) solutions of different concentrations were prepared using 95\% ethanol as solvent [14]. Total content of flavonoids is given as equivalent content of rutin in dry raw material.

High performance liquid chromatography (HPLC) method was used for identification and quantification of steviol glycosides and hydroxycinnamic acids. The analysis was performed using an automatic four-channel liquid chromatograph Agilent 1100 with diode-matrix detector and chemical analyzer (Agilent Technologies, Germany). For preparation of the extracts for analysis, air-dried leaves or callus were finely chopped, poured over with methanol in the $1 \mathrm{~g}: 20 \mathrm{~mL}$ ratio, kept for $24 \mathrm{~h}$ in the dark and filtered using dense $(0.2 \mu \mathrm{m})$ teflon filter. For estimating the quantity of steviol glycosides and hydroxycinnamic acids, the areas of appropriate signals were compared with the ones for reference substances (stevioside, rebaudioside, caffeic acid).

For preparation of the AgNPs, $9 \mathrm{~mL}$ of $1 \mathrm{mM} \mathrm{AgNO}_{3}$ (Merck) aqueous solution were added to $1 \mathrm{~mL}$ of extract. The Extract $/ \mathrm{AgNO}_{3}$ reaction mixture was stirred for 2 hours at temperature of $40^{\circ} \mathrm{C}$, then the solution was cooled to room temperature and the UV spectra of the obtained colloid were recorded. For all the Extract/AgNO reaction mixtures, changing the colour of the mixtures from yellow to brown, which indicates the formation of AgNPs, was observed in about 30 minutes after beginning of the reaction.

UV/Vis spectra of plant extracts and AgNPs colloids were recorded on a Perkin Elmer Lambda 35 $\mathrm{UV} / \mathrm{Vis}$ double beam Spectrophotometer at $25^{\circ} \mathrm{C}$ in the wavelength range of 200-800 nm. Scanning speed was $480 \mathrm{~nm} / \mathrm{min}$, cuvette path length was $10 \mathrm{~mm}$.

The antioxidant activity of the extracts and AgNPs colloids was evaluated using 2,2-diphenyl-1-picrylhydrazyl (DPPH) radical scavenging method [15]. A $0.15 \mathrm{mM}$ solution of DPPH (Sigma-Aldrich) in 70\% ethanol was prepared. To $2 \mathrm{~mL}$ of the DPPH solution $2 \mathrm{~mL}$ of $70 \%$ ethanol and $1 \mathrm{~mL}$ of original or diluted extract/colloid were consecutively added. The concentration of stable radicals in reaction mixture was determined from the change in optical density at the absorption maximum of $520 \mathrm{~nm}$. For characterization of the antioxidant ability of the extracts and colloids, percentages of the reduced DPPH radicals for the reaction time of $30 \mathrm{~min}$ (DPPH-30 values) were determined. For diluted Stevia extracts, the kinetics of radical inhibition during 2 hours was also investigated.

To characterize antimicrobial properties of the extracts and colloids, we studied their ability to inhibit the growth of the test culture in a standard meat-peptone broth (MPB) medium, with Bacillus cereus cells as a test culture. A volume of $4 \mathrm{~mL}$ of MPB, $0.5 \mathrm{~mL}$ of extract/colloid (original or diluted by 2-10 times) and $0.5 \mathrm{~mL}$ of Bacillus cereus cells suspension were placed in a test tube, the concentration of the cells in the reaction medium was $0.5 \mathrm{million}$ cells $/ \mathrm{mL}$. Reference samples were prepared by the same way, but $0.5 \mathrm{~mL}$ of extract/colloid was substituted for $0.5 \mathrm{~mL}$ of MPB or $0.5 \mathrm{~mL}$ of ethanol solution of different concentration. The content of tubes was mixed and incubated at $30^{\circ} \mathrm{C}$ for $24 \mathrm{~h}$. The concentration of Bacillus cereus cells in the suspension was determined from UV spectra, using the concentrationdependent absorbance of Bacillus cereus cells at $\lambda=540 \mathrm{~nm}$. Percentage of the inhibited by extracts/colloids cells were calculated as a difference in concentration of Bacillus cereus cells in the studied suspension and control samples. The presented results are the average of three independent measurements.

\section{Results and discussion}

Table 1 shows the total phenol index, equivalent concentration of ascorbic acid and results of DPPH-30 test for Stevia extracts prepared from the leaves of the plants grown ex situ and in vitro and from callus culture (Extract 1-3, respectively). Table 2 gives the data regarding the content of phytoconstituents with antioxidant 
and antimicrobial properties in the Stevia raw material. Three main groups of bioactive compounds were revealed in the plants and extracts: steviol glycosides, flavonoids and hydroxycinnamic acids. Flavonoids and hydroxycinnamic acids are phenolic compounds with high redox properties, while steviol glycosides are known to possess distinctive antimicrobial activity. In addition to the above-mentioned compounds, small amounts of fatty acids, reducing sugars and terpenoids were also detected in the extracts during our previous experiment by means of laser desorption/ionization mass spectrometry method.

Table 1

Antioxidants properties of Stevia extracts.

\begin{tabular}{ccccc}
\hline $\begin{array}{c}\text { Raw material used for extract } \\
\text { preparation }\end{array}$ & $\begin{array}{c}\text { Extract } \\
\text { designation }\end{array}$ & $\begin{array}{c}\text { Total phenol } \\
\text { index }\end{array}$ & $\begin{array}{c}\text { Equivalent concentration }{ }^{*} \text { of } \\
\text { ascorbic acid, mM }\end{array}$ & DPPH-30*, \% \\
\hline Leaves of plant grown ex situ & Extract 1 & $10.8 \pm 0.2$ & $5.4 \pm 0.1$ & $\sim 100$ \\
Leaves of plant grown in vitro & Extract 2 & $4.0 \pm 0.1$ & $2.0 \pm 0.1$ & $\sim 100$ \\
Callus & Extract 3 & $8.0 \pm 0.1$ & $4.0 \pm 0.1$ & $\sim 100$ \\
\hline
\end{tabular}

${ }^{*}$ Indicated scattering of the data corresponds to $90 \%$ confidence interval

Table 2

Content of phytoconstituents in the Stevia raw material.

\begin{tabular}{cccc}
\hline Raw material used for extract preparation & \multicolumn{3}{c}{ Content of bioactive compounds ${ }^{*} \%$} \\
\cline { 2 - 3 } & Flavonoids & Steviol glycosides & Hydroxycinnamic acids \\
\hline Leaves of plant grown ex situ & $0.92 \pm 0.05$ & $2.8 \pm 0.1$ & $1.0 \pm 0.1$ \\
Leaves of plant grown in vitro & $0.60 \pm 0.05$ & $1.9 \pm 0.1$ & $3.3 \pm 0.1$ \\
Callus & $0.11 \pm 0.05$ & $\leq 0.1$ & $8.3 \pm 0.1$ \\
\hline
\end{tabular}

*Indicated scattering of the data corresponds to $90 \%$ confidence interval

As one can see from the Tables 1 and 2, all the extracts have high content of phenols, which is equivalent to a concentration of ascorbic acid of 2-5 mM. The highest content of phenols, flavonoids, and steviol glycosides were observed for the leaves of the grown ex situ plants and for the appropriate Extract 1. Callus and Extract 3 are characterized by the highest content of hydroxycinnamic acids and by significant value of total phenol index, the content of flavonoids and steviol glycosides being negligible. As to the plants grown in vitro, they contain the smallest amount of phenols and intermediate amount of flavonoids and steviol glycosides.

All original extracts were found to possess antioxidant ability that was sufficient to inhibit practically $100 \%$ of DPPH radicals for 30 minutes (Table 1). To reveal the distinction in the antioxidant properties of the extracts, the DPPH test was also performed using the diluted by 200 times extracts (Figure 1). As one can conclude from the Figure 1, the activity of diluted extracts in reaction with DPPH radicals during $2 \mathrm{~h}$ is represented by the series: Extract $1 \approx$ Extract $3>>$ Extract 2 . Thus, antioxidant activity of the extracts correlates with total phenols content, rather than flavonoids content (Table 1). At the same time, composition of the extracts appears to contribute to the reaction kinetics: high amount of flavonoids in Extract 1 leads to faster reduction of DPPH radicals during first 10 min of the reaction, as compared to Extract 3 (Figure 1). Antimicrobial properties of the extracts are illustrated by Table 4 data and will be discussed below.

All the extracts revealed significant activity in the AgNPs synthesis. Figure 2 gives the UV spectra for Extract/ $\mathrm{AgNO}_{3}$ reaction mixtures in $2 \mathrm{~h}$ after the start of the reaction. As one can see from the Figure 2, all the spectra include the absorbance band with the maximum at around $450 \mathrm{~nm}$, which is characteristic of AgNPs plasmon resonance. The smallest intensity of the band is characteristic for Extract $2 / \mathrm{AgNO}_{3}$ reaction mixture and close to each other are the bands for Extract $1 / \mathrm{AgNO}_{3}$ and Extract $3 / \mathrm{AgNO}_{3}$ mixtures. The different efficiency of the extracts in the synthesis of AgNPs may be explained by the distinctions in the extracts composition; as one can conclude from the comparison of Figures 1 and 2, this efficiency correlates with the antioxidant activity of the extracts, which was determined by using DPPH test. Indeed, Extract 2 is characterized by the smallest content of phenols and by the lowest rate of DPPH inhibition; it also has the less activity in AgNPs synthesis. Extract 1 has the highest content of phenols and flavonoids, which are very effective redox agents [16], and during first two hours of the reactions, shows the fastest reduction of both DPPH radicals and $\mathrm{Ag}^{+}$ions. Extract 3 from the callus culture is similar to Extract 1 by its redox properties and exhibits the similar activity in AgNPs synthesis.

Table 3 gives the data on antioxidant and antimicrobial properties for AgNPs colloids that were synthesized using Stevia leaves extracts. As it was above-mentioned, all original extracts possessed antioxidant ability sufficient to inhibit $\sim 100 \%$ of DPPH radicals under standard DPPH-30 test conditions. The same observation appears to be true for the appropriate colloids: in spite of partial consumption of reducing agents for the reduction of $\mathrm{Ag}^{+}$ions, all colloids still possess high antioxidant ability and practically reduce $100 \%$ of DPPH radicals for $30 \mathrm{~min}$ (Table 3 ). All colloids are also very effective antimicrobial agents inhibiting $80-85 \%$ of Bacillus cereus cells. 


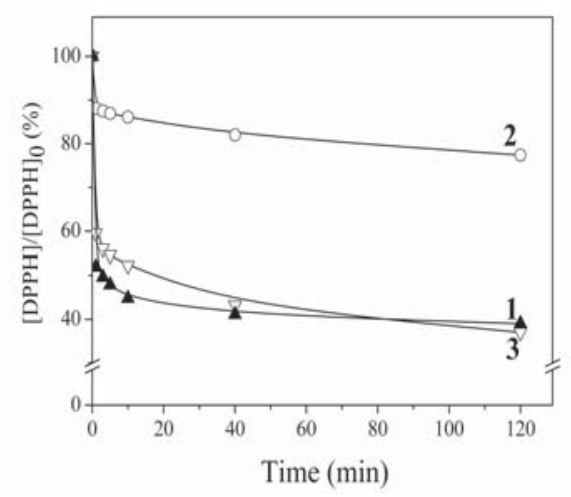

Figure 1. Inhibition of DPPH radicals by diluted Stevia extracts.

(1)-Extract 1 from the leaves of plants grown ex situ;

(2)-Extract 2 from the leaves of plants grown in vitro; (3)-Extract 3 from callus culture.

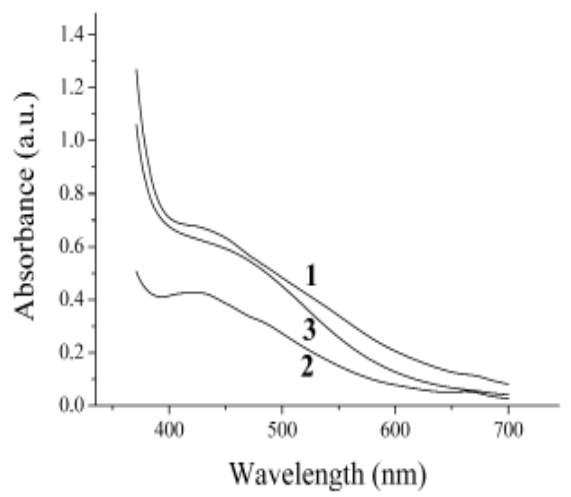

Figure 2. UV spectra of Extract/AgNO ${ }_{3}$ reaction mixtures.

(1)-Extract 1/AgNO ; (2) - Extract 2/AgNO;

(3)-Extract 3/AgNO. Time of reaction: $2 \mathrm{~h}$.

Table 3

Antioxidant and antimicrobial properties of AgNPs colloids.

\begin{tabular}{|c|c|c|c|}
\hline \multirow{2}{*}{$\begin{array}{c}\text { Raw material used for extract } \\
\text { preparation }\end{array}$} & \multirow[t]{2}{*}{ Colloid designation } & \multicolumn{2}{|c|}{ Colloid characteristics } \\
\hline & & $D P P H-30^{*}, \%$ & $\begin{array}{c}\text { Inhibition* of } \\
\text { Bacillus cereus cells, \% }\end{array}$ \\
\hline Leaves of plant grown ex situ & Extract 1/ AgNPs colloid & $\sim 100$ & $85 \pm 7$ \\
\hline Leaves of plant grown in vitro & Extract 2/ AgNPs colloid & $\sim 100$ & $80 \pm 7$ \\
\hline Callus & Extract 3/ AgNPs colloid & $\sim 100$ & $80 \pm 5$ \\
\hline
\end{tabular}

${ }^{*}$ Indicated scattering of the data corresponds to $90 \%$ confidence interval

Table 4 gives the data on antimicrobial properties for diluted Stevia extracts and for appropriate AgNPs colloids. In general, antimicrobial properties of Extract/AgNPs colloids must be a result of antimicrobial action both of extract and silver ions/NPs. However, antimicrobial properties of ethanol extracts may be caused by action of both of extract constituents and ethanol itself.

Antimicrobial properties of diluted colloids, extracts, and ethanol solutions.

\begin{tabular}{|c|c|c|c|c|}
\hline Sample & $\begin{array}{l}\text { Raw material used for extract / } \\
\text { colloid preparation }\end{array}$ & $\begin{array}{l}\text { Dilution, } \\
\text { times }\end{array}$ & $\begin{array}{c}\text { Percentages } \\
\text { of ethanol in } \\
\text { solution/ extract/ } \\
\text { colloid }\end{array}$ & $\begin{array}{c}\text { Percentages }{ }^{*} \text { of } \\
\text { cells inhibited by } \\
\text { solution/ extract/ } \\
\text { colloid }\end{array}$ \\
\hline $70 \%$ ethanol & - & 10 & 7 & 0 \\
\hline $70 \%$ ethanol & - & 5 & 14 & $20 \pm 4$ \\
\hline $70 \%$ ethanol & - & 2 & 35 & $63 \pm 5$ \\
\hline Extract 1 & Leaves of plant grown ex situ & 2 & 35 & $60 \pm 8$ \\
\hline Extract 2 & Leaves of plant grown in vitro & 2 & 35 & $65 \pm 7$ \\
\hline Extract 3 & Callus & 2 & 35 & $58 \pm 5$ \\
\hline Extract 1 & Leaves of plant grown ex situ & 5 & 14 & $30 \pm 5$ \\
\hline Extract 2 & Leaves of plant grown in vitro & 5 & 14 & $35 \pm 6$ \\
\hline Extract 3 & Callus & 5 & 14 & $23 \pm 5$ \\
\hline Extract 1 & Leaves of plant grown ex situ & 10 & 7 & 0 \\
\hline Extract 2 & Leaves of plant grown in vitro & 10 & 7 & 0 \\
\hline Extract 3 & Callus & 10 & 7 & 0 \\
\hline Extract 1/AgNPs colloid & Leaves of plant grown ex situ & 2 & 3.5 & $70 \pm 3$ \\
\hline Extract 2/AgNPs colloid & Leaves of plant grown in vitro & 2 & 3.5 & $65 \pm 7$ \\
\hline Extract 3/AgNPs colloid & Callus & 2 & 3.5 & $60 \pm 7$ \\
\hline Extract 1/AgNPs colloid & Leaves of plant grown ex situ & 5 & 1.4 & $55 \pm 3$ \\
\hline Extract 2/AgNPs colloid & Leaves of plant grown in vitro & 5 & 1.4 & $55 \pm 3$ \\
\hline Extract 3/AgNPs colloid & Callus & 5 & 1.4 & $50 \pm 7$ \\
\hline
\end{tabular}

*Indicated scattering of the data corresponds to $90 \%$ confidence interval 
The data of Table 4 show that the twice-diluted extracts suppress 58-65\% of the Bacillus cereus cells, while ethanol in the appropriate concentration inhibits the same percentages of the cells. Five-fold diluted extracts suppressed cells growth to a higher extent than five-fold diluted 70\% ethanol (23-35\% cells suppressed instead of 20\%). Thus, the difference of 3-15\% may be attributed to antimicrobial effect of the extracts constituents. The stronger inhibition of Bacillus cereus cells by diluted Extract 1 and Extract 2 (Table 4) appears to occur due to the higher content of constituents, such as: steviol, glycosides and flavonoids, which are known to possess high antimicrobial activity [17-19]. Since original extracts in Extract/AgNPs colloids are diluted by ten times and ten-fold diluted extracts practically did not suppress the growth of cells (Table 4), antimicrobial properties of the colloids are caused by inhibiting effect of silver ions/NPs. All colloids exhibited similar antimicrobial activity with the distinctions being about the accuracy of measurements.

\section{Conclusions}

Leaves of Stevia plants grown ex situ and in vitro as well as callus culture formed on damaged leaves were found to be valuable raw material for extraction of bioactive compounds with antioxidant and/or antimicrobial properties. Steviol glycosides, hydroxycinnamic acids and flavonoids were the main groups of bioactive compounds revealed in the extracts, with the content of compounds and properties of the extracts being dependent on the type of raw material.

All extracts have demonstrated high redox properties and significant activity in the synthesis of AgNPs. The activity of the extracts in the reactions with both DPPH radicals and $\mathrm{Ag}^{+}$ions correlated with their total phenol content and changed in the series: Extract from the leaves of plants grown ex situ $\approx$ Extract from callus culture $>>$ Extract from the leaves of plants grown in vitro.

All extracts suppressed the growth of Bacillus cereus cells, the effect being more significant for the extracts from the leaves of plants grown ex situ and in vitro with the higher content of steviol glycosides and flavonoids. All colloids were very effective antimicrobial agents, seemingly due to the action of AgNPs and still possessed high antioxidant properties.

Thus, Stevia rebaudiana extracts and Extract/AgNPs colloids showed both antioxidant and antimicrobial activity, the properties of extracts and colloids being dependent on the type of raw material used for extracts/colloids preparation. The high redox properties of the extract from callus culture tissue indicate a great potential of biotechnological methods for production of pure bioactive compounds and extracts for biological and medical applications.

\section{References}

1. Brewer, M.S. Natural antioxidants: Sources, compounds, mechanisms of action, and potential applications. Comprehensive Reviews in Food Science and Food Safety, 2011, 10, pp. 221-247.

2. Gurib-Fakim, A. Medicinal plants: Traditions of yesterday and drugs of tomorrow. Molecular Aspects of Medicine, 2006, 27, pp. 1-93.

3. Shahid-ul-Islam; Shahid, M.; Mohammad, F. Perspectives for natural product based agents derived from industrial plants in textile applications - a review. Journal of Cleaner Production, 2013, 57, pp. 2-18.

4. Lupascu, L.; Rudic, V.; Cotos, V.; Lupascu, T. Antimicrobial activity of the autochthonous compound Enoxil. Journal of Biomedical Science and Engineering, 2010, 3, pp. 758-762.

5. Cowan, M.M. Plant products as antimicrobial agents. Clinical Microbiology Reviews, 1999, 12(4), pp. 564-582.

6. Malhotra, B.; Keshwani, A.; Kharkwal, H. Antimicrobial food packaging: potential and pitfalls. Frontiers in Microbiology, 2015, 6, pp. 611-620.

7. Herman, A.; Herman, A.P.; Domagalska, B.W.; Młynarczyk, A. Essential oils and herbal extracts as antimicrobial agents in cosmetic emulsion. Indian Journal of Microbiology, 2013, 53(2), pp. 232-237.

8. Iravani, S. Green synthesis of metal nanoparticles using plants. Green Chemistry, 2011, 13, pp. 2638-2650.

9. Goyal, S.; Samsher, S.; Goyal, R. Stevia (Stevia rebaudiana) a bio-sweetener: a review. International Journal of Food Sciences and Nutrition, 2010, 61, pp. 1-10.

10. Gupta, E.; Purwar, S.; Sandaram, S.; Gai, G.K. Nutritional and therapeutic values of Stevia rebaudiana: A review. Journal of Medicinal Plants Research, 2013, 7, pp. 3343-3353.

11. Komarova, M.N.; Nikolaeva, L.A.; Regir, V.G. Phytochemical analysis of medicinal plants: guidelines for laboratory studies, St. Petersburg: State Chemical-Pharmaceutical Academy, 1998, 60 p. (in Russian).

12. Alonso, A.M.; Domianguez, C.; Guillean, D.; Barroso, C.G. Determination of antioxidant power of red and white wines by a new electrochemical method and its correlation with polyphenolic content. Journal of Agricultural and Food Chemistry, 2002, 50, pp. 3112-3115.

13. Stavinskaya, O.M.; Kuzema, P.O.; Laguta, I.V.; Pakhlov, E.M.; Kazakova, O.O.; Chernyavskaya, T.V. Interaction of ascorbic acid with hydrophilic-hydrophobic silicas. Annales Universitatis Mariae Curie-Sklodowska, 2007, 62, pp. 124-135. 
14. Andreeva, V.Yu.; Kalinkina, G.I. Development of method of quantitative determination of flavonoids in Alchemilla vulgaris. Khimiva rastitel'nogo syr'ya, 2000, 1, pp. 85-88 (in Russian).

15. Brand-Williams, W.; Cuvelier, M.E.; Berset, C. Use of a free radical method to evaluate antioxidant activity. LWT - Food Science and Technology, 1995, 28(1), pp. 25-30.

16. Rice-Evans, C.A.; Miller, N.J.; Paganga, G. Structure-antioxidant activity relationships of flavonoids and phenolic acids. Free Radical Biology and Medicine, 1996, 20, pp. 7933-7956.

17. Puri, M.; Sharma, D. Antibacterial activity of stevioside towards food-borne pathogenic bacteria. Engineering in Life Sciences, 2011, 11, pp. 326-329.

18. Cushnie, T.P.T.; Lamb, A.J. Antimicrobial activity of flavonoids. International Journal of Antimicrobial Agents, 2005, 26, pp. 343-56.

19. Desbois, A.P.; Smith, V.J. Antibacterial free fatty acids: activities, mechanisms of action and biotechnological potential. Applied Microbiology and Biotechnology, 2010, 85, pp. 1629-1642. 